\title{
Numerical Investigation of Thermal Counterflow of He II Past Cylinders
}

\author{
Cyprien Soulaine, ${ }^{1,2}$ Michel Quintard, ${ }^{2}$ Bertrand Baudouy, ${ }^{3}$ and Rob Van Weelderen ${ }^{4}$ \\ ${ }^{1}$ Stanford University, Energy Resources Engineering, 367 Panama St, Stanford, California 94305-2220, USA \\ ${ }^{2}$ Institut de Mécanique des Fluides de Toulouse (IMFT)_Université de Toulouse, CNRS-INPT-UPS, 31400 Toulouse, France \\ ${ }^{3}$ CEA Saclay, Irfu/SACM, F-91191 Gif-sur-Yvette, France \\ ${ }^{4}$ CERN, CH-1211 Geneva 23, Switzerland
}

(Received 3 August 2016; published 17 February 2017)

\begin{abstract}
We investigate numerically, for the first time, the thermal counterflow of superfluid helium past a cylinder by solving with a finite volume method the complete so-called two-fluid model. In agreement with existing experimental results, we obtain symmetrical eddies both up- and downstream of the obstacle. The generation of these eddies is a complex transient phenomenon that involves the friction of the normal fluid component with the solid walls and the mutual friction between the superfluid and normal components. Implications for flow in a more realistic porous medium are also investigated.
\end{abstract}

DOI: 10.1103/PhysRevLett.118.074506

Ten years ago, in a paper published in Nature Physics, Zhang and Van Sciver [1] reported interesting and unexpected experimental observations about thermal counterflow of superfluid helium (He II). They used a cryogenic particle image velocimetry (PIV) technique to obtain direct visualization of quantum turbulence past a cylinder in an experimental setup commonly used in fluid mechanics to investigate flow structures around an obstacle. It consisted of a vertical Hele-Shaw cell that contains a solid cylinder in the middle. The cell was initially saturated with $\mathrm{He}$ II and its top side was connected to an He II bath. The bottom of the cell was heated to generate the superfluid counterflow: the normal fluid component flows from the heater to the bath while the superfluid component moves in countercurrent. Compared to classical fluids for which turbulent structures appear downstream of the cylinder, this experiment has shown two apparently stationary eddies downstream of the cylinder but also two large vortices in front of the obstacle. This complex behavior was observed later by different research teams worldwide with an analog experimental setup $[2,3]$. No clear explanations of the phenomena that lead to these recirculations and their apparent stability have been proposed yet and research in this area is still a hot topic. A first attempt of explanation for the apparent stability of such eddies has been proposed by Sergeev and Barenghi [4] entirely from the viewpoint of classical fluid dynamics without any references to quantum turbulence. However, Chagovets and Van Sciver [2] have shown experimentally that below a certain critical velocity corresponding to the onset of quantum turbulence, the normal fluid flow appears as a classical laminar flow with

Published by the American Physical Society under the terms of the Creative Commons Attribution 3.0 License. Further distribution of this work must maintain attribution to the author(s) and the published article's title, journal citation, and DOI. two symmetrical eddies downstream of the cylinder. Clearly, this suggests that the quantum turbulence, usually described as a mutual friction between the normal and superfluid components, plays a key role in the generation of these complex structures.

It must be emphasized that the reasons to investigate quantum turbulence go beyond these fundamental experiments. Indeed, it provides a very challenging theoretical problem that might help to improve our comprehension of turbulence in general [5-7]. From an engineering perspective, a good understanding of the underlying physics of superfluid helium flows will help to improve the heat and mass transfer modeling for cryogenics engineering applications. In particular, Soulaine et al. [8] suggested that the mutual friction during forced flow of He II can yield to a temperature increase of the same order of magnitude than the one resulting from the Joule-Thomson effect only. Clearly, the origin of this additional temperature increase comes from quantum turbulence, and new insights in this research area will help to clarify this statement.

Superfluid helium flow past obstacles is a first step towards the development of a complete theory of superfluid flow in porous media. This topic is of significant interest for cryogenic engineering devices cooled with He II, such as, for instance, the porous structures involved in superconducting high field magnets for high energy particle accelerators or porous plugs in space applications $[9,10]$. When dealing with porous materials, the interactions at the solid boundaries become predominant and an accurate description of the physics at the pore level would help to have an idea of the large scale governing equations. It has been demonstrated that for very low velocity values, i.e., when the mutual friction is negligible and the streamlines embrace the solid structure, the flow can be modeled by a Darcy-like problem $[11,12]$. When increasing slightly the normal fluid flow rate, the streamlines are deformed by the inertia effect [2] and, by analogy with classical fluids, we may expect a nonlinear 
deviation to Darcy's law known as the Forchheimer correction [13]. What is the situation for higher velocities when quantum turbulence arises? Are the eddies similar to the pure Navier-Stokes problem? Are they confined in the pore space as is believed to be the case for classical fluids [14] at least up to a certain Reynolds number [15], and, in this case, can a Darcy-Forchheimer formalism model these nonlinearities, or are there larger scale turbulence effects that require a more complex model? Direct numerical simulations of superfluid flow in a porous medium model can shed some light on these fundamental questions.

In this work, we use our recently developed simulator, HellFOAM [16], to numerically reproduce the experiments of superfluid flow past solid obstacles. HellFOAM solves the complete two-fluid model for superfluid helium [17-19] in a Eulerian grid. In this model, He II is seen as a mixture of different miscible fluids. One of these is a normal viscous fluid (denoted by subscript $n$, and the viscosity is $\mu_{n}$ ), the other is a superfluid (with subscript $s$ ) that moves with zero viscosity. The actual density of He II, $\rho=\rho_{n}+\rho_{s}$, is defined as the sum of the normal and superfluid density, $\rho_{n}$ and $\rho_{s}$, respectively. These quantities depend on the temperature: $\rho_{s}$ vanishes at the so-called $\lambda$ point where the fluid becomes fully normal and $\rho_{n}$ is null at absolute zero. The mass conservation for the whole fluid reads

$$
\frac{\partial \rho}{\partial t}+\nabla \cdot\left(\rho_{n} \mathbf{v}_{n}+\rho_{s} \mathbf{v}_{s}\right)=0
$$

The velocity field of the normal $\left(\mathbf{v}_{n}\right)$ and superfluid $\left(\mathbf{v}_{s}\right)$ components are governed by a set of two coupled equations. The momentum balance equation for the normal component reads (gravity and diffusion energy effects neglected)

$$
\begin{aligned}
\frac{\partial \rho_{n} \mathbf{v}_{n}}{\partial t}+\nabla \cdot\left(\rho_{n} \mathbf{v}_{n} \mathbf{v}_{n}\right)= & -\frac{\rho_{n}}{\rho} \nabla p-\rho_{s} s \nabla T+\nabla \cdot\left(\mu_{n} \nabla \mathbf{v}_{n}\right) \\
& -A \rho_{n} \rho_{s}\left|\mathbf{v}_{n}-\mathbf{v}_{s}\right|^{2}\left(\mathbf{v}_{n}-\mathbf{v}_{s}\right),
\end{aligned}
$$

and for the superfluid component reads

$$
\begin{aligned}
\frac{\partial \rho_{s} \mathbf{v}_{s}}{\partial t}+\nabla \cdot\left(\rho_{s} \mathbf{v}_{s} \mathbf{v}_{s}\right)= & -\frac{\rho_{s}}{\rho} \nabla p+\rho_{s} s \nabla T \\
& +A \rho_{n} \rho_{s}\left|\mathbf{v}_{n}-\mathbf{v}_{s}\right|^{2}\left(\mathbf{v}_{n}-\mathbf{v}_{s}\right) .
\end{aligned}
$$

Beside these two momentum conservation equations, an energy equation is considered where the entropy $s$ is transported by the normal fluid only. We have

$$
\frac{\partial \rho s}{\partial t}+\nabla \cdot\left(\rho s \mathbf{v}_{n}\right)=\frac{A \rho_{n} \rho_{s}\left|\mathbf{v}_{n}-\mathbf{v}_{s}\right|^{4}}{T} .
$$

In Eqs. (2) and (3), $\rho_{s} s \nabla T$ represents the thermomechanical force that occurs when a temperature gradient exists. It is responsible for creating the counterflow in which the normal fluid moves down the temperature gradient from the heat source to the bath, while the superfluid flows toward the heat source. The dissipative term $A \rho_{n} \rho_{s}\left|\mathbf{v}_{n}-\mathbf{v}_{s}\right|^{2}\left(\mathbf{v}_{n}-\mathbf{v}_{s}\right)$, which has an influence in the equations only at high velocities, represents the mutual friction term as introduced by Gorter and Mellink [20,21] to model the interaction between the two components when the superfluid velocity reaches a certain critical value. $A$ is a coefficient determined empirically. The last term on the right-hand side of Eq. (4) expresses the energy dissipation due to the mutual friction between the two components. We note that, besides the mutual friction term, Eqs. (1)-(4) are strongly coupled by the presence of the temperature gradient in the momentum equations. Additionally, the physical properties $s$ and $A$ depend on temperature, which also contributes to the coupling of the equations. The fluid properties $\rho_{n}, \rho_{s}, \mu_{n}$, $s$, and $A$ are provided by polynomial interpolations from the HePak thermodynamic database by Cryodata, Inc. [22].

The dimensionless forms of Eqs (2)-(4) are [23]

$$
\begin{gathered}
\left(\frac{\partial \rho_{n}^{\prime} \mathbf{v}_{n}^{\prime}}{\partial t^{\prime}}+\nabla^{\prime} \cdot\left(\rho_{n}^{\prime} \mathbf{v}_{n}^{\prime} \mathbf{v}_{n}^{\prime}\right)\right) \\
=-\frac{1}{a} \frac{\rho_{n}^{\prime}}{\rho^{\prime}} \nabla^{\prime} p^{\prime}-\rho_{s}^{\prime} s^{\prime} \nabla^{\prime} T^{\prime}+\frac{1}{\Re} \nabla^{\prime} \cdot\left(\mu_{n}^{\prime} \nabla^{\prime} \mathbf{v}_{n}^{\prime}\right) \\
\quad-\gamma A^{\prime} \rho_{n}^{\prime} \rho_{s}^{\prime}\left|\mathbf{v}_{n}^{\prime}-\mathbf{v}_{s}^{\prime}\right|^{2}\left(\mathbf{v}_{n}^{\prime}-\mathbf{v}_{s}^{\prime}\right) \\
\left(\frac{\partial \rho_{s}^{\prime} \mathbf{v}_{s}^{\prime}}{\partial t^{\prime}}+\nabla^{\prime} \cdot\left(\rho_{s}^{\prime} \mathbf{v}_{s}^{\prime} \mathbf{v}_{s}^{\prime}\right)\right)= \\
-\frac{\rho_{s}^{\prime}}{\rho^{\prime}} \nabla^{\prime} p^{\prime}+a \rho_{s}^{\prime} s^{\prime} \nabla^{\prime} T^{\prime} \\
+a \gamma A^{\prime} \rho_{n}^{\prime} \rho_{s}^{\prime}\left|\mathbf{v}_{n}^{\prime}-\mathbf{v}_{s}^{\prime}\right|^{2}\left(\mathbf{v}_{n}^{\prime}-\mathbf{v}_{s}^{\prime}\right)
\end{gathered}
$$

and

$$
\left(\frac{\partial \rho^{\prime} s^{\prime}}{\partial t^{\prime}}+\nabla \cdot\left(\rho^{\prime} s^{\prime} \mathbf{v}_{n}^{\prime}\right)\right)=\gamma \frac{A^{\prime} \rho_{n}^{\prime} \rho_{s}^{\prime}\left|\mathbf{v}_{n}^{\prime}-\mathbf{v}_{s}^{\prime}\right|^{4}}{T^{\prime}} .
$$

Theses equations involve three dimensionless parameters: the ratio of densities, $a=\left(\rho_{s}^{0} / \rho_{n}^{0}\right)$, the Reynolds number
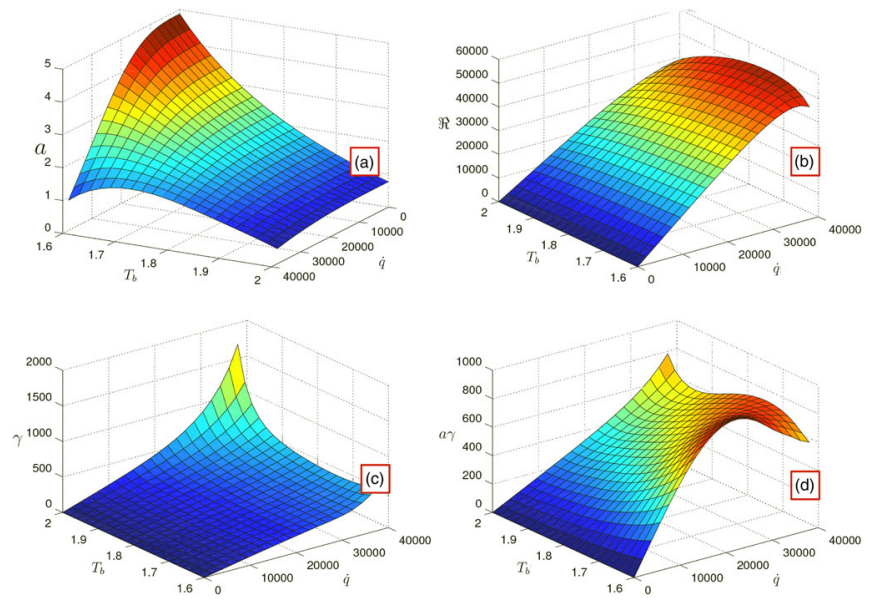

FIG. 1. Variation of the dimensionless numbers with heat flux and bath temperature, (a) densities ratio $a$, (b) Reynolds number $\Re$, (c) mutual friction over the advection of the normal component $\gamma$, and (d) mutual friction over the advection of the superfluid component $a \gamma$. 
$\Re=\left(\rho_{n}^{0} v_{n}^{0} d / \mu_{n}^{0}\right)$, where $d$ is a characteristic length and another dimensionless number, $\gamma$, that quantifies the mutual friction over the advection of the normal component. This latter is defined as

$$
\gamma=A^{0} \rho_{s}^{0}\left(1+\frac{\rho_{n}^{0}}{\rho_{s}^{0}}\right)^{3} v_{n}^{0} d=A^{0} \mu_{n}^{0} \frac{(1+a)^{3}}{a^{2}} \Re .
$$

Dimotakis [24] proposed a dimensionless number analog to $\gamma$ and showed that it is the appropriate scale for the mutual friction in pure counterflow. Our many numerical tests confirm Dimotakis's assertion and show that the different flow patterns observed up- and downstream the cylinder are not governed by the Reynolds number as in classical fluid mechanics but mainly by $\gamma$, i.e., by the friction term. In these dimensionless numbers, all physical parameters with superscript " 0 " are evaluated from HePak at the reference temperature $T^{0}=T_{b}+\Delta T$, where $T_{b}$ is the bath temperature and $(\Delta T / L)=\left(A \rho_{n} / \rho_{s}^{3} s^{4} T^{3}\right) \dot{q}^{3}$ is the gradient induced by the heat flux [25]. The dimensionless velocity is the one imposed by the heat flux, i.e., $\dot{q}=\rho^{0} s^{0} T^{0} v_{n}^{0}$. Variations of $a, \Re, \gamma$, and $a \gamma$ with heat flux and bath temperature are plotted in Fig. 1. If the evolution of the Reynolds number is quasilinear with the heat flux increase as illustrated by Fig. 1(b), the variation of $a$ and $\gamma$ are not always monotonic: for example, for low bath temperature such as $T_{b}=1.6 \mathrm{~K}$, ar reaches a maximum for $\dot{q}=$ $22 \mathrm{~kW} / \mathrm{m}^{2}$ and decreases for higher heat fluxes. The Reynolds number, which essentially depends linearly on $\dot{q}$, will play a role similar to the one it plays for classical viscous fluids, i.e., the sequence of hydrodynamic instabilities from laminar flow, to downstream vortices and eventually full turbulence. Since the two velocity fields have very different topological features due to the different boundary conditions at the walls, the friction term will generate vorticity which will be large if $a \gamma$ is large enough [notice the complex interplay between $a$ and $\gamma$ in Fig. 1(d)].

In our simulator, HellFOAM, the dimensional superfluid equations are solved on a Eulerian grid and discretized with the finite volume method. The coupling is handled with a sequential algorithm called Super-PISO. For extensive details on the algorithm and its implementation refer to Soulaine et al. [16]. The implementation has been validated by comparison of the numerical results with the analytical solutions of superfluid flow in a capillary for different flow regimes [16]. We also used the simulator to reproduce forced flow experiments [26]. The agreement is good in the sense that the temperature evolution along the tube is captured correctly with our numerical model regardless the mass flow rate [8].

In this work, HellFOAM is used to reproduce experiments of thermal counterflow of superfluid helium past a cylinder. The geometry is a two-dimensional vertical channel, $200 \mathrm{~mm}$ long, $16 \mathrm{~mm}$ wide, and a $6.35 \mathrm{~mm}$ diam solid cylinder located in the middle. On the solid walls, a no-slip boundary condition for the normal fluid and a slip

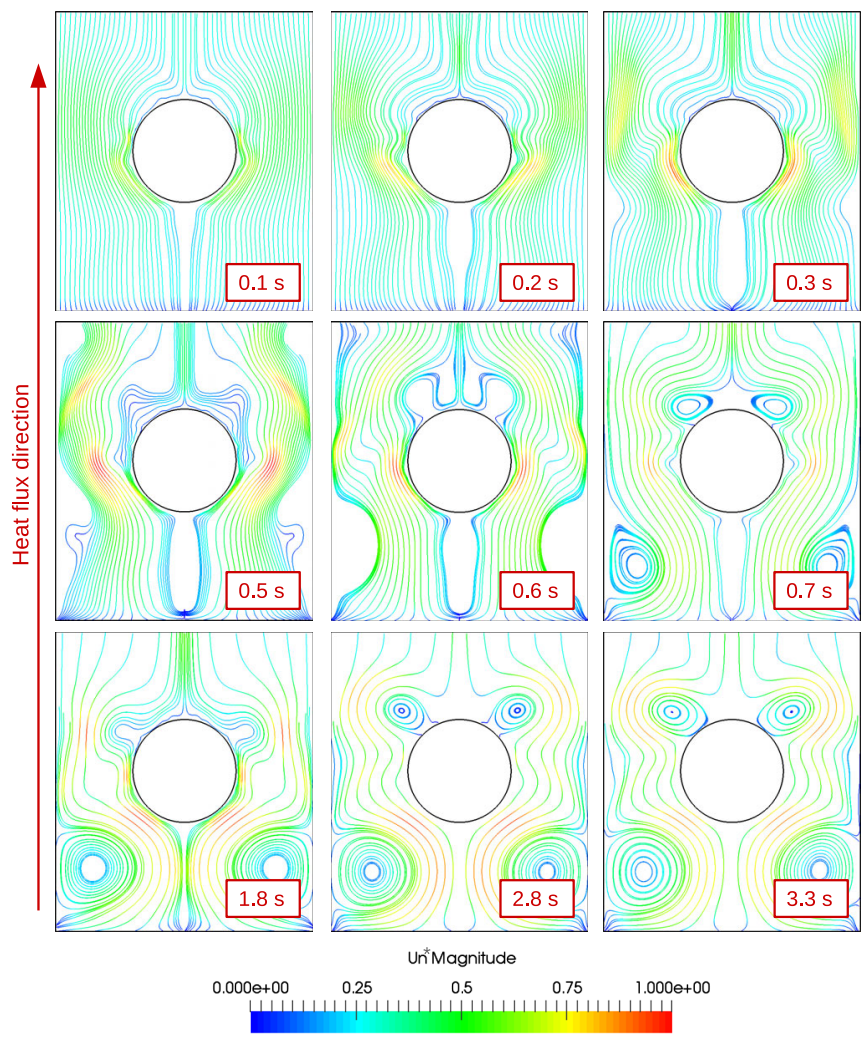

FIG. 2. Streamlines of the normal fluid component past a cylinder at early time steps.

condition for the superfluid component are considered. The solid surfaces are adiabatic and the heater is located at the bottom side. Several simulations for various heat flux densities $\left(\dot{q}=0.5\right.$ to $\left.37 \mathrm{~kW} / \mathrm{m}^{2}\right)$ and bath temperatures $\left(T_{b}=1.6\right.$ to $\left.2.0 \mathrm{~K}\right)$ have been performed. In Fig. 2 we have plotted the streamlines at the early time steps for the normal component for $T_{b}=2.0 \mathrm{~K}$ and $\dot{q}=12.5 \mathrm{~kW} / \mathrm{m}^{2}$. The sequence that leads to the up- and downstream quasistationary eddies is the result of complex transient mechanisms. Just after the heater is turned on, the normal components flow towards the bath entrance with streamlines parallel to the solid walls like a classical creeping flow. Very quickly, the viscous forces for the normal component increase in the boundary layers adjacent to the solid walls of both the cylinder and the channels and eventually generates eddies downstream the solid obstacle. Since the superfluid components flow at counter-current with a slip condition at the walls, the mutual friction in the boundary layers increases as well, which generates a source force in the momentum equations and then triggers the vortices upstream the cylinder. All the simulations reach a quasisteady state at about 3 to $4 \mathrm{sec}$ after the heater was turned on. The velocity patterns of the normal flow are in good agreement with the PIV measurements of Zhang and Van Sciver: we observe two symmetrical eddies downstream of the cylinder between the cylinder and the channel walls, and two other, larger, recirculations upstream. At long times, the upstream eddies are very stable while the 

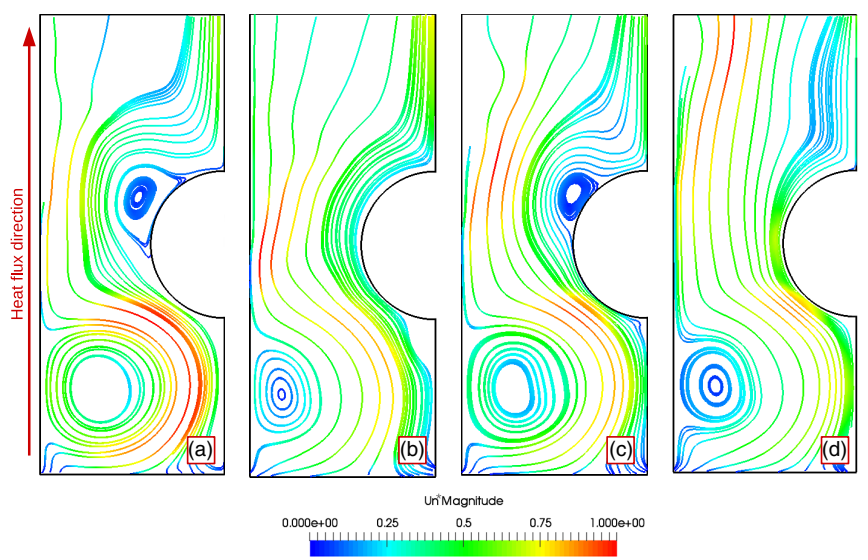

FIG. 3. Streamlines of the normal fluid component. (a) $T_{b}=$ $2.0 \mathrm{~K}, \dot{q}=25 \mathrm{~kW} / \mathrm{m}^{2}, \gamma=620, a=0.68$; (b) $T_{b}=1.8 \mathrm{~K}$, $\dot{q}=25 \mathrm{~kW} / \mathrm{m}^{2}, \quad \gamma=300, \quad a=1.88 ; \quad$ (c) $T_{b}=1.9 \mathrm{~K}, \dot{q}=$ $37 \mathrm{~kW} / \mathrm{m}^{2}, \gamma=633, a=0.97$; (d) $T_{b}=1.6 \mathrm{~K}, \dot{q}=25 \mathrm{~kW} / \mathrm{m}^{2}$, $\gamma=288, a=3.1$.

downstream ones are sensitive to some fluctuations. All the simulations we ran displayed these large stable upstream recirculations. As illustrated in Fig. 3 for the steady state, their size is correlated with $\gamma$ : the higher the value, the larger the eddies' diameter. Not all our simulations, however, show downstream eddies. It appears, as shown in Figs. 3(a), 3(c), that the instabilities generated in the boundary layer remain persistent only if $a<1$, i.e., when the normal fluid density is higher than the density of the superfluid component. Compared to classical fluid mechanics, superfluid couplings play a dominant role, with wave propagation at low values of a induced by the thermomechanical force and dominant fluid interaction effects due to the mutual friction force as $a \gamma$ increases. For very low heat fluxes, the mutual friction term is negligible and the normal flow is only coupled with the superfluid component through the thermomechanical term, $\rho_{s} s \nabla T$. We were not able, however, to recover the classic hydrodynamics pattern as observed experimentally [2] because our model does not reproduce well the bath entrance boundary condition, and a heat wave was propagating into the domain with many reflections, generating an unsteadystate pattern. The pure Navier-Stokes situations are only recovered for the limit case $\rho_{s} \rightarrow 0$, with the classical sequence (i) $\Re \sim 15$ (for the geometry in use) appearance of symmetrical, steady-state downstream vortices, (ii) $\Re \sim 1300$, appearance of unsteady, nonsymmetrical vortices, then more and more turbulent flows.

Analysis of the flow structures past a cylinder is only the first step towards a better understanding of the different regimes of superfluid flow in porous media. Our above results suggest that the interaction terms may impact dramatically the velocity topology. This is illustrated here using a two-dimensional domain made of circular grains with a polydispersed size distribution [27]. Figure 4 represents the computational domain we used for the simulations. It consists of a $6 \times 17 \mathrm{~mm}^{2}$ square domain containing 48 cylinders with diameters ranging from 0.5 to $2 \mathrm{~mm}$ that leads to a porosity of $30 \%$ and a numerically estimated permeability of $10^{-9} \mathrm{~m}^{2}$. The void space is gridded with $2 \times 10^{5}$ hexahedral cells. The pore space is initially filled with $\mathrm{He}$ II at $1.6 \mathrm{~K}$ and the right-hand side is connected to the bath. The left-hand side is then warmed up with a heat flux density $\dot{q}=0.14 \mathrm{~kW} / \mathrm{m}^{2}(a=4.8$, $\gamma=0.28)$ and $\dot{q}=14 \mathrm{~kW} / \mathrm{m}^{2}(a=4.2, \gamma=28)$, respectively. These fluxes lead to a temperature elevation of 0.01 and $330 \mathrm{mK}$, respectively. The velocity profiles obtained for the normal component are plotted in Figs. 4(a), 4(b). As for the case of superfluid helium counterflow past a cylinder, we observe two distinct flow regimes: (i) for low heat flux, the flow patterns are similar to laminar flow in classical hydrodynamics in porous media, (ii) beyond a certain threshold that remains to be determined precisely, quantum turbulence arises and streamlines indicate large vortices in the pore spaces. To emphasize the role played by the Gorter-Mellink term, we run the same simulation
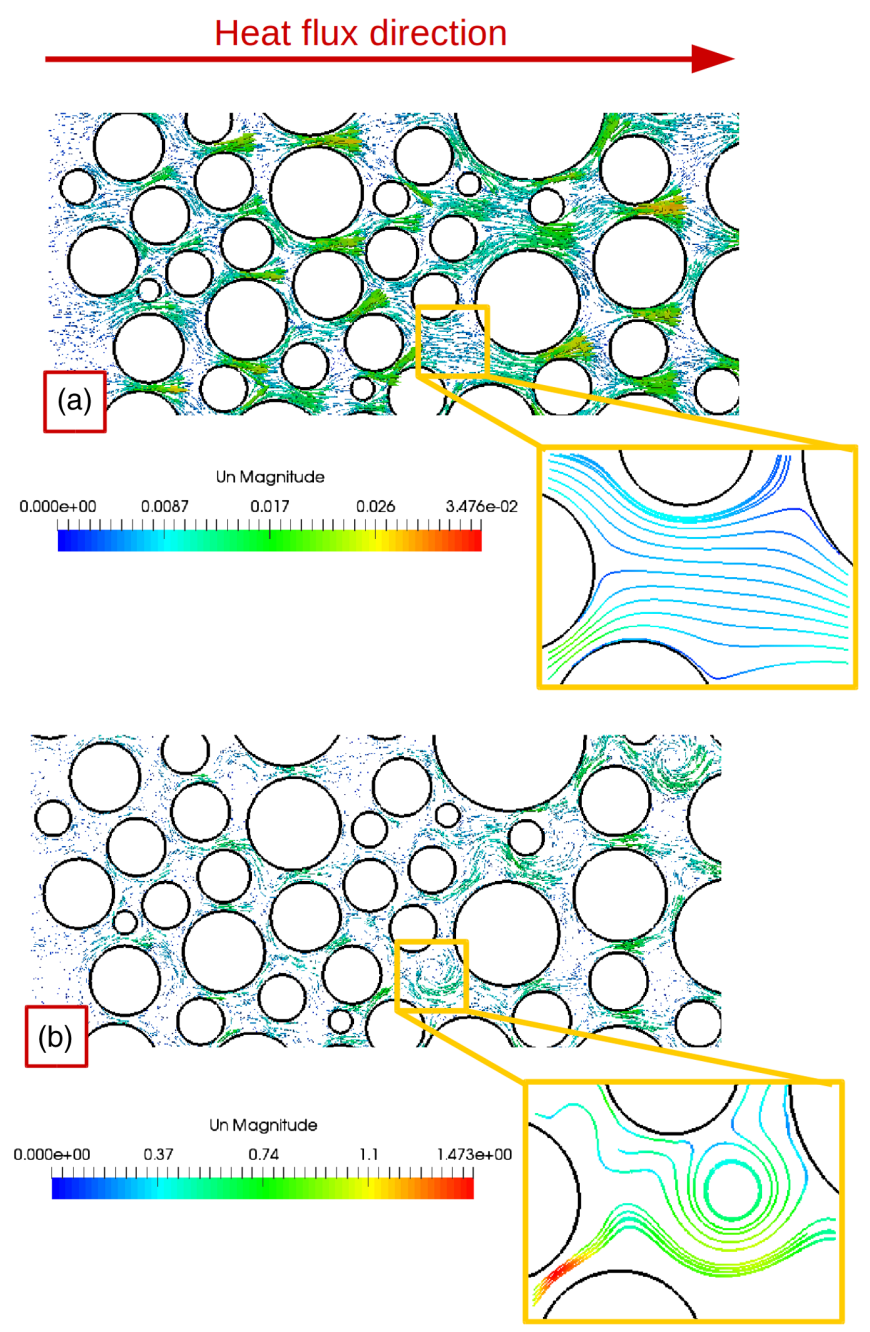

FIG. 4. Velocity vectors and streamlines for the normal component. (a) At low heat flux the flow pattern is similar to laminar flow in classical hydrodynamics. (b) For high heat flux, large vortices appear in the pore space. 
turning this term off. In such a case, the simulation results do not show any vortex. This confirms that these complex vortices are generated indeed by the mutual friction term. Regarding the question of Darcy-scale modeling, it is noteworthy that the vortices remain in the intrapore space and that eddies spanning over several pores are not observed, at least for the range of parameters studied. This is reminiscent of other studies of turbulent flow in classical hydrodynamics in porous media [14] and suggests that a law of a Darcy-Forchheimer type can be used to simulate the flow in such flow regimes.

Based on numerical simulations of counterflow of superfluid helium past a cylinder, using the two-fluid model with mutual friction forces between the normal and superfluid particles, we can draw the following conclusions. Our simulation results are in good qualitative agreement with the different PIV experiments of the literature. In particular, we were able to capture the four quasisteady large eddies, both up- and downstream of the cylinder. These flow patterns are clearly linked to the impact of the mutual friction term in the two-fluid model, as controlled by the two proposed dimensionless numbers. Interestingly, these results also suggest that the two-fluid model with mutual friction terms contains all the necessary physics to explain the formation of these eddies. Beyond the agreement of these initially surprising experimental observations with the modeling, these results also illustrate the potential complexity of such flows in porous media. More work must be done, but this kind of simulation will certainly be valuable to get more insights about an adequate theory of superfluid flow in porous media, which in turn would offer direct applications to sensitive technological problems such as high field magnets cooled with superfluid helium or porous plug-in space applications.

[1] T. Zhang and S. W. Van Sciver, Large scale turbulent flow around a cylinder in counterflow superfluid ${ }^{4} \mathrm{He}$ (He II), Nat. Phys. 1, 36 (2005).

[2] T. V. Chagovets and S. W. Van Sciver, Visualization of He II counterflow around a cylinder, Phys. Fluids 25, 105104 (2013).

[3] D. Duda, M. La Mantia, M. Rotter, and L. Skrbek, On the visualization of thermal counterflow of He II past a circular cylinder, J. Low Temp. Phys. 175, 331 (2014).

[4] Y. A. Sergeev and C. F. Barenghi, Normal fluid eddies in the thermal counterflow past a cylinder, J. Low Temp. Phys. 156, 268 (2009).

[5] Russell J. Donnelly, Superfluid turbulence, Sci. Am. 259, 100 (1988).

[6] L. Skrbek and K. R. Sreenivasan, Developed quantum turbulence and its decay, Phys. Fluids 24, 011301 (2012).

[7] W. F. Vinen, Quantum turbulence: Aspects of visualization and homogeneous turbulence, J. Low Temp. Phys. 175, 305 (2014).

[8] C. Soulaine, M. Quintard, H. Allain, B. Baudouy, and R. Van Weelderen, Numerical investigation of heat transfer in a forced flow of He II, in Proceedings of the 15th International Heat Transfer Conference, IHTC-15 August 10-15, 2014, Kyoto, Japan, 2014.

[9] P. P. Granieri, Heat transfer through cable insulation of $\mathrm{Nb}-\mathrm{Ti}$ superconducting magnets operating in $\mathrm{He}$ II, Cryogenics 53, 61 (2013).

[10] H. Allain, R. Van Weelderen, B. Baudouy, M. Quintard, M. Prat, and C. Soulaine, Investigation of suitability of the method of volume averaging for the study of heat transfer in superconducting accelerator magnet cooled by superfluid helium, Cryogenics 53, 128 (2013).

[11] H. Allain, M. Quintard, M. Prat, and B. Baudouy, Upscaling of superfluid helium flow in porous media, Int. J. Heat Mass Transfer 53, 4852 (2010).

[12] H. Allain, B. Baudouy, M. Quintard, and M. Prat, Experimental investigation of heat transfer through porous media in superfluid helium, Cryogenics 66, 53 (2015).

[13] P. Forchheimer, Wasserbewegung durch boden, Z. Ver. Deutsch. Ing. 45, 1782 (1901).

[14] C. Soulaine and M. Quintard, On the use of a DarcyForchheimer like model for a macro-scale description of turbulence in porous media and its application to structured packings, Int. J. Heat Mass Transfer 74, 88 (2014).

[15] Y. Jin, M.-F. Uth, A. V. Kuznetsov, and H. Herwig, Numerical investigation of the possibility of macroscopic turbulence in porous media: A direct numerical simulation study, J. Fluid Mech. 766, 76 (2015).

[16] C. Soulaine, M. Quintard, H. Allain, B. Baudouy, and R. Van Weelderen, A PISO-like algorithm to simulate superfluid helium flow with the two-fluid model, Comput. Phys. Commun. 187, 20 (2015).

[17] L. Tisza, Transport phenomena in helium II, Nature (London) 141, 913 (1938).

[18] L. Landau, Theory of the superfluidity of helium II, Phys. Rev. 60, 356 (1941).

[19] I. M. Khalatnikov, An Introduction to the Theory of Superfluidity (W. A. Benjamin, New York, 1965).

[20] C. J. Gorter and J. H. Mellink, On the irreversible processes in liquid helium-II, Physica (Amsterdam) 15, 285 (1949).

[21] C. J. Gorter, P. W. Kasteleijn, and J. H. Mellink, Discussion of the properties of helium-II on the basis of the two fluid model, Physica (Amsterdam) 16, 113 (1950).

[22] Cryodata Inc., Hepak, version 3.4, P.O. Box 173, Louisville, CO 80027, http://www.htess.com/hepak.htm.

[23] See Supplemental Material at http://link.aps.org/ supplemental/10.1103/PhysRevLett.118.074506 for a numerical investigation of thermal counterflow of He-II past cylinders.

[24] P. E. Dimotakis, Gorter-mellink scale, and critical velocities in liquid-helium-II counterflow, Phys. Rev. A 10, 1721 (1974).

[25] V Arp, Heat transport through helium II, Cryogenics 10, 96 (1970).

[26] S. Fuzier and S. W. Van Sciver, Experimental measurements and modeling of transient heat transfer in forced flow of He II at high velocities, Cryogenics 48, 130 (2008).

[27] P. De Anna, T. Le Borgne, M. Dentz, A. M. Tartakovsky, D. Bolster, and P. Davy, Flow intermittency, dispersion, and correlated continuous time random walks in porous media, Phys. Rev. Lett. 110, 184502 (2013). 